\title{
Isolation of domains of stability of linear dynamic systems with fractional regulators
}

\author{
Oleksii Lobok ${ }^{1}$, Borys Goncharenko', \\ Larysa Vykhrova², Maryna Sych ${ }^{3}$
}

\section{1 - National University of Food Technologies, Kyiv, Ukraine \\ 2 - Central Ukrainian National Technical University, Kropivnitsky, Ukraine \\ 3 - National University of Bioresources and Nature Use, Kyiv, Ukraine}

\section{Keywords:}

Stability

Fractional

Derivative

Integral

Controller

D-split

Dipereintegrator

Article history:

Received

21.10.2017

Received in revised

form 01.11.2017

Accepted

29.12.2017

\section{Corresponding}

author:

Borys Goncharenko

E-mail:

GoncharenkoBN

@i.ua

\section{Abstract}

Introduction. The research of linear dynamic systems of regulation of the process of biological purification of contaminated water with fractional regulators was conducted in order to determine the boundaries of $D$ - domains of their global stability and to determine the space of the parameters of the adjustment of the fractional controller for fixed orders of the diereintegrators in its composition.

Materials and methods. Studies were conducted on the stability of the automatic control of the water treatment process. Using the $D$ split method, we obtain analytical formulas that determine the limits of the region of stable stabilization of the "object" + "fractionalregulator" system.

Result and discussion. Automatic control systems of fractional order are more precisely described by dynamic equations, in which the order of derivatives can be any number, valid and not only integer. Proportional-integral-differential (PID-) regulators that are widely used in practice of automation also fall under a fractional generalization, if in their structure, instead of ordinary integer derivatives and integrals, fractional analogues are used. The controllers of the fractional order denote how $P I^{\lambda} D^{\mu}$, where $\lambda$ and $\mu$ are the orders of integration and differentiation of the error signal, with orders $\lambda$ and $\mu$ may have valid non-integer (fractional) values.

On the basis of the $D$-split method, analytical formulas are described that describe the boundaries of global stability of linear dynamic systems of fractional order. Domains of stability are built in the space of parameters of the configuration of fractional domains $P I^{\lambda} D^{\mu}$-regulators for fixed orders of dipintegrators. An appropriate algorithmic software is developed that implements the proposed method for selecting the domain of stability. Some results of computational experiments are given, an estimation of fractional $P I^{\lambda} D^{\mu}$ - regulator efficiency is given.

Conclusions. On the basis of the D-split method, analytical expressions were obtained, which describe the boundaries of the global region of stability of linear dynamic systems of fractional order of type "input-output" with fractional $P I^{\lambda} D^{\mu}$-regulators.

DOI:

$10.24263 / 2310$

$1008-2017-5-2-12$ 


\section{Introduction}

From the beginning of the development of the theory of integro-differential calculus of fractional order [1], its first applications in control problems appeared only about 50 years ago [2]. It has been shown that fractional calculus becomes an effective tool for describing numerous dynamic systems. The classical results of the PID control theory have spread to the fractional order controllers, which denote how $P I^{\lambda} D^{\mu}$, where $\lambda$ and $\mu$ are the orders of integration and differentiation of the error signal, with orders $\lambda$ and $\mu$ may have valid non-integer (fractional) values $[3,4]$.

\section{Materials and methods}

Studies were conducted on the stability of the automatic control of the water treatment process. Analytical studies are based on Laplace's transformation with respect to the operator of a dipintegrator, which generalizes the functions of differentiation and integration, on the use of the structural theory of closed systems and on the target mathematical transformations of the transfer functions of the object-regulator system.

On the basis of the $D$-split method, analytical formulas are described that describe the boundaries of global stability of linear dynamic systems of fractional order.

Domains of stability are built in the space of parameters of the configuration of fractional domains $P I^{\lambda} D^{\mu}$-regulators for fixed orders of dipintegrators.

An appropriate algorithmic software is developed that implements the proposed method for selecting the domain of stability.

Analytical studies and computational experiment in the MATLAB environment have been conducted. Using the $D$-split method, we obtain analytical formulas that determine the limits of the region of stable stabilization of the "object" + "fractional-regulator" system.

\section{Result and discussion}

The well-known problem of the allocation of the global region of stability ( $D$-split method) required the distribution of fractional dynamic systems in the space of the parameters of the adjustment $P I^{\lambda} D^{\mu}$-regulator, depending on the value of the orders of powers $\lambda$ and $\mu$.

The purpose of the article is to study the possibility of applying the $D$-split method to automatic control systems for process control with fractional controllers.

A fundamental operator ${ }_{a} D_{t}^{\gamma}$ is often referred to as a differintegrator.

$$
{ }_{a} D_{t}^{\gamma}= \begin{cases}d^{\gamma} / d t^{\gamma}, & \gamma>0, \\ 1, & \gamma=0, \\ \int_{a}^{t}(d \tau)^{-\gamma}, & \gamma<0\end{cases}
$$

where $\gamma$ - fractional order, $a$-constant associated with the initial conditions. 
More fundamental is the definition of Grunwald-Letnikov for the order $\gamma$ according to which

$$
{ }_{a} D_{t}^{\gamma} f(t)=\lim _{h \rightarrow 0} \frac{1}{h^{\gamma}} \sum_{j=0}^{[(t-a) / h]}(-1)^{j}\left(\begin{array}{l}
\gamma \\
j
\end{array}\right) f(t-j h),
$$

where $\left(\begin{array}{l}\gamma \\ j\end{array}\right)=\frac{\Gamma(\gamma+1)}{\Gamma(j+1) \Gamma(\gamma-j+1)}, \Gamma(x)-$ gamma Euler's function, $h>0-$ gain of the time coordinate, $f(x)$ - the function to which the operator of the differential integration is used, $[\cdot]$ - means an integer part of the number. This definition shows that integer derivatives require the use of finite series, and fractional derivatives - an infinite number of members of a series.

It can be proved [5] that the Laplace transform, which is the basis of the definition of the concept of a transfer function, for the differintegrator has the form

$$
L\left\{{ }_{0} D_{t}^{\gamma} f(t)\right\}=\int_{0}^{\infty} e^{-s t} D_{t}^{\gamma} f(t) d t=s^{\gamma} F(s)-\left.\sum_{j=0}^{n-1} s^{j}(-1)^{j}{ }_{0} D_{t}^{\gamma-j-1} f(t)\right|_{t=0},
$$

where $F(s)=L\{f(t)\}$ - ordinary Laplace transform function $f(x), n$ - an integer that satisfies the condition $n-1<\gamma \leq n$. Note that if $\left.{ }_{0} D_{t}^{\gamma-j-1} f(t)\right|_{t=0}=0, j=0,1,2, \ldots, n-1$, then from (3) it follows that $L\left\{{ }_{0} D_{t}^{\gamma} f(t)\right\}=s^{\gamma} F(s)$. Systems with fractional orders have transfer functions of arbitrary real order.

Consider the transfer function of fractional order, which is given by the following expression

$$
G(s)=\frac{N(s)}{D(s)}=\frac{b_{n} s^{\beta_{n}}+b_{n-1} s^{\beta_{n-1}}+\ldots+b_{1} s^{\beta_{1}}+b_{0} s^{\beta_{0}}}{a_{n} s^{\alpha_{n}}+a_{n-1} s^{\alpha_{n-1}}+\ldots+a_{1} s^{\alpha_{1}}+a_{0} s^{\alpha_{0}}}=\frac{\sum_{i=0}^{n} b_{i} s^{\beta_{i}}}{\sum_{i=0}^{n} a_{i} s^{\alpha_{i}}}
$$

where $a_{i}, b_{i}, \beta_{n}>\beta_{n-1}>\ldots>\beta_{1}>\beta_{0} \geq 0, \alpha_{n}>\alpha_{n-1}>\ldots>\alpha_{1}>\alpha_{0} \geq 0$ - arbitrary valid numbers.

In the time domain, the transfer function corresponds to an inhomogeneous differential equation of the fractional order of the form

$$
\sum_{i=0}^{n} a_{i} D^{\alpha_{i}} y(t)=\sum_{i=0}^{n} b_{i} D^{\beta_{i}} u(t)
$$

where $y(t)$ - exit, and $u(t)$ - input of the control object, ${ }_{a} D_{t}^{\gamma}-$ differintegrator.

In the general structure of the closed control system of fractional order with one input and one output is presented $y(t)$ - output, $r(t)$ - input request signal, $e(t)$ - error (mismatch), $u(t)$ - control signal, $G(s)$ - transfer function of the control object, $C(s)-$ transfer function of the fractional order controller. 
Transmission function of the fractional $P I^{\lambda} D^{\mu}{ }_{\text {_ }}$ controller has the form

$$
C(s)=k_{p}+k_{i} s^{-\lambda}+k_{d} s^{\mu},
$$

where $\lambda$ и $\mu$-fractional orders whose values belong to the region $(0,2), k_{p}, k_{i}, k_{d}$ - adjusting parameters of the regulator.

In the time domain, the transfer function (6) corresponds to the type control

$$
u(t)=k_{p} \cdot e(t)+k_{i} \cdot\left({ }_{0} D_{t}^{-\lambda} e(t)\right)+k_{d} \cdot\left({ }_{0} D_{t}^{\mu} e(t)\right),
$$

where $-{ }_{0} D_{t}^{\gamma}$ differintegrator.

The task is to find the area of stability with allowable values of the settings $k_{p}, k_{i}, k_{d}$ fractional $P I^{\lambda} D^{\mu}$ - controller, which stabilize the control object. This is important when designing $P I^{\lambda} D^{\mu}$ - controllers, and then in the future and to find optimal regulators on the found parametric area of stabilization by the chosen criterion.

Transmission function of the system "object + regulator" in Fig. 1 has the form

$$
W(s)=\frac{C(s) G(s)}{1+C(s) G(s)}=\frac{Q(s)}{P(s)},
$$

where

$$
\begin{gathered}
Q(s)=\sum_{j=0}^{n}\left[k_{p} b_{j} s^{\lambda+\beta_{j}}+k_{i} b_{j} s^{\beta_{j}}+k_{d} b_{j} s^{\lambda+\mu+\beta_{j}}\right], \\
P(s)=\sum_{j=0}^{n}\left[a_{j} s^{\lambda+\alpha_{j}}+k_{p} b_{j} s^{\lambda+\beta_{j}}+k_{i} b_{j} s^{\beta_{j}}+k_{d} b_{j} s^{\lambda+\mu+\beta_{j}}\right] .
\end{gathered}
$$

The area of stable stabilization, which we denote through $S$, in the space of parameters is subject to belonging to the left half-plane of the complex $s$ - plane all real parts of the roots of the characteristic quasipolynomial $P(s)$, which for convenience will be presented in the form

$$
P(s)=\sum_{j=0}^{n} p_{j} s^{q_{j}}=p_{n} s^{q_{n}}+p_{n-1} s^{q_{n-1}}+\ldots+p_{1} s^{q_{1}}+p_{0} s^{q_{0}},
$$

where $q_{j}$ - ordered fractional orders of powers, and moreover $q_{n}>q_{n-1}>\ldots>q_{0}$, $p_{j}$ - coefficients determined by the factors of the transfer function of the control object and the parameters of the settings $k_{p}, k_{i}, k_{d}$ fractional $P I^{\lambda} D^{\mu}$ - regulator.

To select the region of stable system stabilization (control object with the controller) we use the $D$-split method, the parameters space [6].

Recall that according to this method, the boundary between the areas of stability and instability in the space of the configuration parameters is three parts: $\Gamma=\Gamma_{0}+\Gamma_{\omega}+\Gamma_{\infty}$. Constituent $\Gamma_{0}$ is determined from the condition of intersection of the real root of the 
characteristic equation of the imaginary axis $s$-plane with $s=0$. That is, the component $\Gamma_{0}$ is found by way of substitution $s=0$ in the equation $P(s)=0$, where $P(s)$ which is determined by the equation (11). It follows that $\Gamma_{0}$ can be determined from the condition $p_{0}=0$, if the value of the smallest order $q_{0}$ equals 0 , i.e. with $s^{q_{0}}=1$. If $q_{0} \neq 0$, i.e. $s^{q_{0}} \neq 1$, then the boundaries $\Gamma_{0}$ does not exist.

Constituent $\Gamma_{\omega}$ is determined from the condition of intersection of a pair of complex conjugate roots of the imaginary axis at $s=j \omega$, where $j=\sqrt{-1}-$ imaginary unit. So, in this case, quasipoline (11) becomes an unstable and valid and imaginary part of the equation $P(j \omega)=0$ begin to equal zero at the same time.

Constituent $\Gamma_{\infty}$ is determined by intersection of the real roots of the quasi-polynomial (11) imaginary axis at $s=\infty$ and can be determined from the condition $p_{n}=0$.

Applying these preconditions to the investigated system "object + regulator" and analyzing the characteristic quasipolin (10), we come to the conclusion that the components $\Gamma_{0}$ and $\Gamma_{\infty}$ the boundaries of the stability zone are straight lines:

$$
\begin{gathered}
\Gamma_{0}-\text { line: }\left\{\begin{array}{l}
k_{i}=0, \quad \text { at } s^{\beta_{0}}=1, \\
\text { not exist, at } s^{\beta_{0}} \neq 1,
\end{array}\right. \\
\Gamma_{\infty}-\text { line: } \begin{cases}k_{d}=0, & \text { at }\left(\alpha_{n}=\beta_{n}\right) \text { aбо }\left(\alpha_{n}>\beta_{n} \text { i } \mu>\alpha_{n}-\beta_{n}\right), \\
k_{d}=-a_{n} / b_{n}, & \text { at }\left(\alpha_{n}>\beta_{n} \text { i } \mu=\alpha_{n}-\beta_{n}\right), \\
\text { not exist, } & \text { at }\left(\alpha_{n}>\beta_{n} \text { i } \mu<\alpha_{n}-\beta_{n}\right) .\end{cases}
\end{gathered}
$$

To build a component $\Gamma_{\omega}$ substitute $s=j \omega$ into the equation $P(s)=0$, where $P(s)$ - quasipolin (10). Then we will get

$$
\begin{aligned}
P(j \omega) & =\sum_{j=0}^{n}\left[a_{j}(j \omega)^{\lambda+\alpha_{j}}+k_{p} b_{j}(j \omega)^{\lambda+\beta_{j}}+k_{i} b_{j}(j \omega)^{\beta_{j}}+k_{d} b_{j}(j \omega)^{\lambda+\mu+\beta_{j}}\right]= \\
& =\operatorname{Re}\{P(j \omega)\}+j \cdot \operatorname{Im}\{P(j \omega)\}=0,
\end{aligned}
$$

where $\operatorname{Re}\{P(j \omega)\}$ and $\operatorname{Im}\{P(j \omega)\}$ mean respectively the actual and imaginary parts of the quasipolin $P(j \omega)$.

For further transformation of the expression (12) we recall that this is not an integer degree of complex number can be calculated by the formula Muavr-Laplace

$$
(\sigma+j \omega)^{\gamma}=\left(\sigma^{2}+\omega^{2}\right)^{\gamma / 2}[\cos (\gamma \varphi)+j \sin (\gamma \varphi)]
$$

where $\varphi=\arctan (\omega / \sigma), \sigma$ - real part, $\omega$ - imaginary part and $\gamma-$ fractional order of a complex number. 
Expression $j^{\gamma}$, which is present in the equation (12), can be presented according to the formula (13), so

$$
j^{\gamma}=\cos \left(\frac{\pi}{2} \gamma\right)+j \sin \left(\frac{\pi}{2} \gamma\right)
$$

Further, equating to zero the real and imaginary part of the equation (12), taking into account the formula (14), we will get

$$
\left\{\begin{array}{l}
\operatorname{Re}\{P(j \omega)\}=k_{p} R_{1 p}(\omega)+k_{i} R_{1 i}(\omega)+k_{d} R_{1 d}(\omega)+H_{1}(\omega)=0, \\
\operatorname{Im}\{P(j \omega)\}=k_{p} R_{2 p}(\omega)+k_{i} R_{2 i}(\omega)+k_{d} R_{2 d}(\omega)+H_{2}(\omega)=0,
\end{array}\right.
$$

where

$$
\begin{array}{cc}
R_{1 p}(\omega)=\sum_{j=0}^{n} b_{j} \omega^{\lambda+\beta_{j}} \cos \left(\frac{\pi}{2}\left(\lambda+\beta_{j}\right)\right), & R_{1 i}(\omega)=\sum_{j=0}^{n} b_{j} \omega^{\beta_{j}} \cos \left(\frac{\pi}{2} \beta_{j}\right), \\
R_{1 d}(\omega)=\sum_{j=0}^{n} b_{j} \omega^{\lambda+\mu+\beta_{j}} \cos \left(\frac{\pi}{2}\left(\lambda+\mu+\beta_{j}\right)\right), & H_{1}(\omega)=\sum_{j=0}^{n} a_{j} \omega^{\lambda+\alpha_{j}} \cos \left(\frac{\pi}{2}\left(\lambda+\alpha_{j}\right)\right), \\
R_{2 p}(\omega)=\sum_{j=0}^{n} b_{j} \omega^{\lambda+\beta_{j}} \sin \left(\frac{\pi}{2}\left(\lambda+\beta_{j}\right)\right), & R_{2 i}(\omega)=\sum_{j=0}^{n} b_{j} \omega^{\beta_{j}} \sin \left(\frac{\pi}{2} \beta_{j}\right), \\
R_{2 d}(\omega)=\sum_{j=0}^{n} b_{j} \omega^{\lambda+\mu+\beta_{j}} \sin \left(\frac{\pi}{2}\left(\lambda+\mu+\beta_{j}\right)\right), & H_{2}(\omega)=\sum_{j=0}^{n} a_{j} \omega^{\lambda+\alpha_{j}} \sin \left(\frac{\pi}{2}\left(\lambda+\alpha_{j}\right)\right) .
\end{array}
$$

The system of linear equations (15) contains more unknowns $\left(k_{p}, k_{i}, k_{d}\right)$, than the number of equations, one of the parameters of the system can be arbitrarily chosen for its unambiguous solution. If as a parameter, choose a coefficient $k_{p}$, then the system (15) becomes a system of linear algebraic equations of the second order with respect to unknowns $k_{i}$ and $k_{d}$, the solution of which has the form

$$
\begin{gathered}
k_{i}=\frac{\Delta_{i}(\omega)}{\Delta(\omega)}, \quad k_{d}=\frac{\Delta_{d}(\omega)}{\Delta(\omega)}, \\
\Delta_{i}(\omega)=R_{1 d}(\omega) H_{2}(\omega)-R_{2 d}(\omega) H_{1}(\omega)+k_{p}\left(R_{1 d}(\omega) R_{2 p}(\omega)-R_{1 p}(\omega) R_{2 d}(\omega)\right), \\
\Delta_{d}(\omega)=R_{2 i}(\omega) H_{1}(\omega)-R_{1 i}(\omega) H_{2}(\omega)+k_{p}\left(R_{1 p}(\omega) R_{2 i}(\omega)-R_{1 i}(\omega) R_{2 p}(\omega)\right), \\
\Delta(\omega)=R_{1 i}(\omega) R_{2 d}(\omega)-R_{1 d}(\omega) R_{2 i}(\omega)=\omega^{\lambda+\mu} \sin \left(\frac{\pi}{2}(\lambda+\mu)\right)\left(R_{1 i}^{2}(\omega)+R_{2 i}^{2}(\omega)\right) .
\end{gathered}
$$

Note that for fractional $P I^{\lambda}$ - regulator $\left(k_{d}=0\right)$ system (15) has a single solution

$$
k_{p}=\frac{\Delta_{p}(\omega)}{\Delta(\omega)}, \quad k_{i}=\frac{\Delta_{i}(\omega)}{\Delta(\omega)},
$$




$$
\begin{gathered}
\Delta_{p}(\omega)=H_{2}(\omega) R_{1 i}(\omega)-H_{1}(\omega) R_{2 i}(\omega), \quad \Delta_{i}(\omega)=H_{1}(\omega) R_{2 p}(\omega)-H_{2}(\omega) R_{1 p}(\omega), \\
\Delta(\omega)=R_{1 p}(\omega) R_{2 i}(\omega)-R_{1 i}(\omega) R_{2 p}(\omega)=-\omega^{\lambda} \sin \left(\frac{\pi}{2} \lambda\right)\left(R_{1 i}^{2}(\omega)+R_{2 i}^{2}(\omega)\right) .
\end{gathered}
$$

We now apply these results to highlight the stability of the biological control system for contaminated water by active sludge with fractional $P I^{\lambda} D^{\mu}$ - regulator. By the assumption that the kinetics of the growth process of biomass is described by the Mono equation [7], in work [8] the linearized model of the bioelectric system "aerotank + sedimentation tank" was obtained in the form of a model with one input and one output.

$$
\frac{d x(t)}{d t}=A x(t)+b u(t), \quad y(t)=x_{2}(t)=c^{T} x(t),
$$

where $\boldsymbol{x}(t)=\left(x_{1}(t), x_{2}(t), x_{3}(t)\right)^{T}-$ state vector in which $x_{1}(t), x_{2}(t)$ - respectively, the concentration of biomass and substrate in aerotanks, $x_{3}(t)$ - the concentration of recirculating biomass from the settling tank to the aerosol bioreactor, $u(t)$ - single-speed control function-speed of dilution (analogue of volume flow rate), $y(t)$ - the observed output of the system is the concentration of the substrate.

System matrix $\boldsymbol{A}$ and vectors $\boldsymbol{b}$ and $\boldsymbol{c}$ are defined as follows

where

$$
\boldsymbol{A}=\left(\begin{array}{lll}
a_{11} & a_{12} & a_{13} \\
a_{21} & a_{22} & a_{23} \\
a_{31} & a_{32} & a_{33}
\end{array}\right), \quad \boldsymbol{b}=\left(\begin{array}{l}
b_{1} \\
b_{2} \\
b_{3}
\end{array}\right), \quad \boldsymbol{c}=\left(\begin{array}{l}
0 \\
1 \\
0
\end{array}\right),
$$

$$
\begin{gathered}
a_{1,1}=\mu_{\max } \frac{x_{2}^{*}}{k_{s}+x_{2}^{*}}-(1+r) u^{*}, \quad a_{1,2}=\mu_{\max } k_{s} \frac{x_{1}^{*}}{\left(k_{s}+x_{2}^{*}\right)^{2}}, \quad a_{1,3}=r u^{*}, \\
a_{2,1}=-\frac{\mu_{\max }}{Y} \frac{x_{2}^{*}}{k_{s}+x_{2}^{*}}, \quad a_{2,2}=-\frac{\mu_{\max } k_{s}}{Y} \frac{x_{1}^{*}}{\left(k_{s}+x_{2}^{*}\right)^{2}}-(1+r) u^{*}, \quad a_{2,3}=0, \\
a_{3,1}=(1+r) u^{*}, \quad a_{3,2}=0, \quad a_{3,3}=-(\beta+r) u^{*}, \\
b_{1}=-(1+r) x_{1}^{*}+r x_{3}^{*}, \quad b_{2}=-(1+r) x_{2}^{*}+s_{i n}, \quad b_{3}=-(\beta+r) x_{3}^{*}+(1+r) x_{1}^{*} .
\end{gathered}
$$

It is marked here: $u^{*}-$ nominal control given, $x^{*}=\left(x_{1}^{*}, x_{2}^{*}, x_{3}^{*}\right)^{T}-$ the corresponding equilibrium state vector calculated for it; $\mu_{\max }$ - maximum specific growth rate of biomass; $k_{s}$ - saturation constant, determined experimentally; $s_{i n}$ - concentration of the substrate in the inlet stream; $Y$ - the factor of output (profitability) of biomass; $r, \beta$-coefficients that determine respectively the ratio of the recirculation flow and biomass waste stream to the input stream.

Numerical simulation of a controlled biocleaning system was carried out at the following output data: $s_{\text {in }}=200[\mathrm{mg} / \mathrm{l}], Y=0.65, \mu_{\max }=0.15\left[\mathrm{~h}^{-1}\right], k_{s}=100[\mathrm{mg} / \mathrm{l}]$, 
$r=0.6, \beta=0.2, u^{*}=0.05\left[h^{-1}\right], t_{0}=0, T=1[h]$, vector of the initial state of the system (20) relied on equal $\boldsymbol{x}^{0}=\left(x_{1}^{0}, x_{2}^{0}, x_{3}^{0}\right)^{T}=(286,17,568)^{T}[\mathrm{mg} / \mathrm{l}]$.

Note that the vector of the equilibrium state of the system (20) with this data was calculated as the solution of the corresponding system of nonlinear equations of the third order and equaled $\boldsymbol{x}^{*}=\left(x_{1}^{*}, x_{2}^{*}, x_{3}^{*}\right)^{T}=(285,15.38,570)^{T}$.

In frequency domain model (20) can be presented in the form

$$
Y(s)=G(s) U(s)
$$

where $U(s), Y(s)$ - Laplace transforms according to input and output, $G(s)$ - transfer function of the control object.

$$
G(s)=c^{T}(s E-A)^{-1} b=\frac{c^{T} \operatorname{adj}(s E-A) b}{\operatorname{det}(s E-A)}=\frac{p_{2} s^{2}+p_{1} s+p_{0}}{s^{3}+q_{2} s^{2}+q_{1} s+q_{0}} .
$$

Here through $\operatorname{adj}(s E-A)$ the matrix attached to the matrix is indicated $s \boldsymbol{E}-\boldsymbol{A}$, and the coefficients $p_{i}, q_{i}$ polynomial numerator and denominator are calculated by the formulas

$$
\begin{gathered}
p_{0}=b_{2} a_{11} a_{33}-b_{1} a_{21} a_{33}-b_{2} a_{13} a_{31}, \quad q_{0}=a_{12} a_{21} a_{33}+a_{13} a_{31} a_{22}-a_{11} a_{22} a_{33}, \\
p_{1}=b_{1} a_{21}-b_{2} a_{11}-b_{2} a_{33}, \quad q_{1}=a_{11} a_{22}+a_{11} a_{33}+a_{22} a_{33}-a_{13} a_{31}-a_{12} a_{21}, \\
p_{2}=b_{2}, \quad q_{2}=-a_{11}-a_{22}-a_{33} .
\end{gathered}
$$

If control $u(t)$ in the time domain construct in a fractional class $P I^{\lambda} D^{\mu}$ - regulators of the form (6)

$$
u(t)=-\left(k_{p} \cdot y(t)+k_{i} \cdot\left({ }_{0} D_{t}^{-\lambda} y(t)\right)+k_{d} \cdot\left({ }_{0} D_{t}^{\mu} y(t)\right)\right)
$$

then the transfer function of the "biocleaning" + "regulator" system will be determined by the expression $W(s)=Q(s) / P(s)$, where $Q(s)=C(s) G(s), P(s)=1+C(s) G(s), C(s)-$ transfer function of the fractional controller, determined by the formula (6), $G(s)$ - transfer function of the control object, calculated by the formulas (21), (22).

To determine the range of valid values for the configuration parameters $k_{p}, k_{i}, k_{d}$ fractional $P I^{\lambda} D^{\mu}$ - regulator, which stabilizes the work of the bio-treatment system, uses the calculated formulas (16), (17) i (18), (19), that describe the boundaries of the stability regions of the system with a fractional controller. Computational experiments were carried out in the MATLAB mathematical system environment. Below are some results from computational experiments. 
In figure 1 in the parameter space $Z=\left\{k_{p}, k_{i}\right\}$ the global region of stability (shaded area) of a bio-waste fractional system is presented $P I^{\lambda}$ - regulator at $\lambda=1$, that is when using the classic $P I$ - regulator $\left(\Gamma_{\omega}, \Gamma_{0}\right.$ - the boundaries of the area of stability).

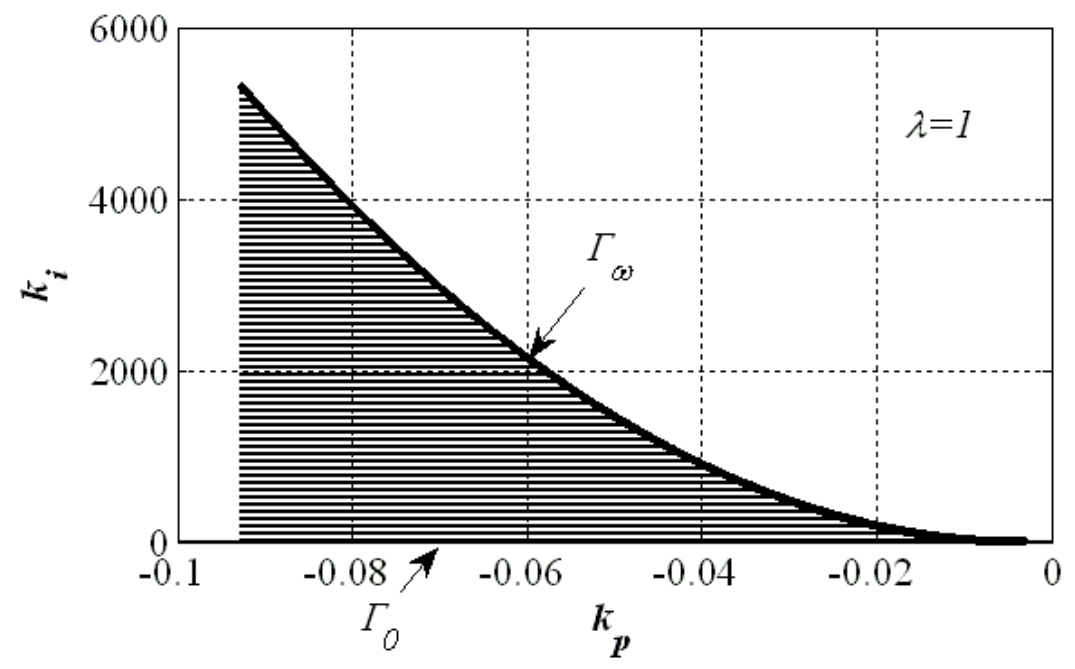

Figure 1. Global region of system stability with $P I^{\lambda}-$ regulator at $\lambda=1$

In figure 2 the areas of stability of the system of bio-purification with fractionation $P I^{\lambda}$-regulator are constructed at different values of the order of the differintegrator.

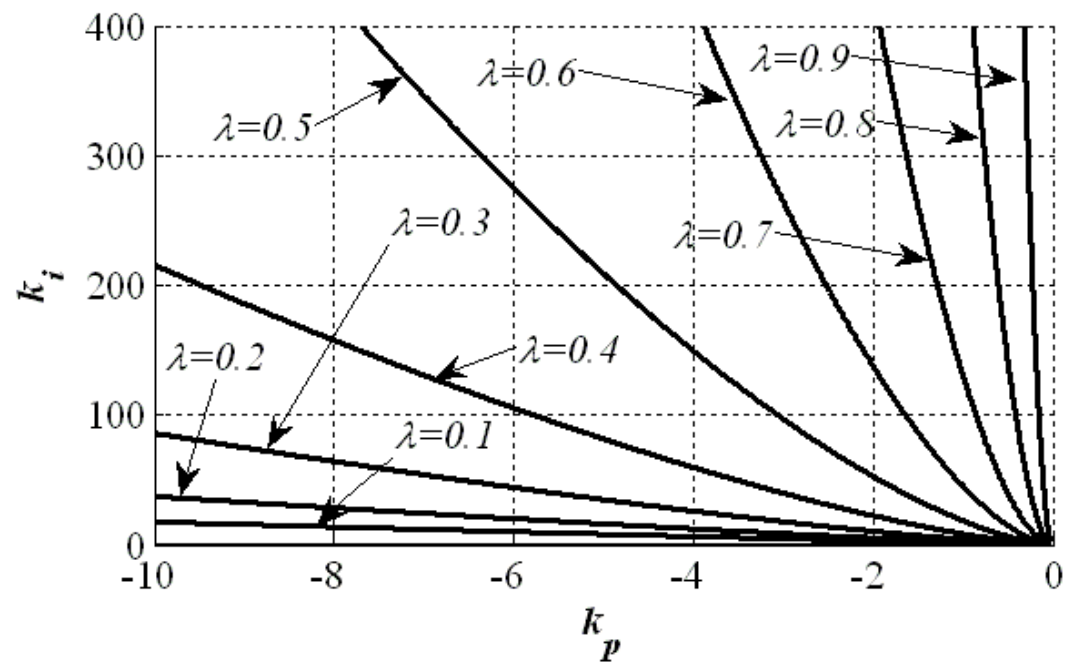

Figure 2. Global areas of stability of the biocleaning system with $P I^{\lambda}$-regulator at $0<\lambda<1$ 


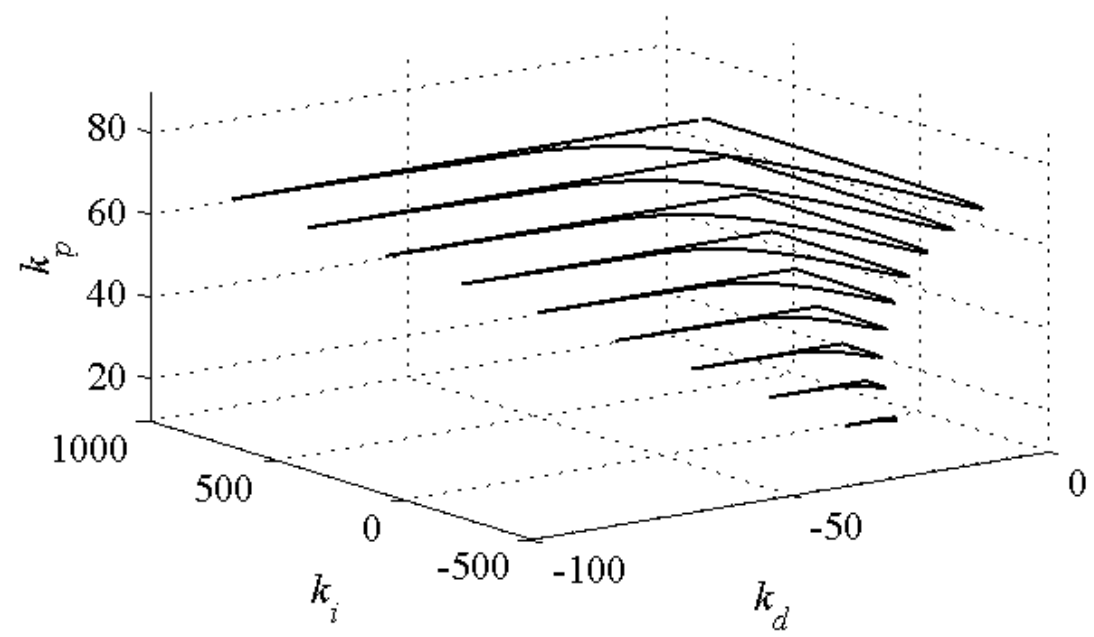

Figure 3. Global areas of stability of the biocleaning system with $3 P I^{\lambda} D^{\mu}$-regulator at $\lambda=0.7, \mu=0.1$

Here, the areas of stability are limited to the bottom of the abscissa, and on the top the curve that matches the value of the order of the parameter. From the graphs it can be seen that with increasing order, the region of stability of the system also increases.

Then studied areas of stability of the system with fractional $P I^{\lambda} D^{\mu}$-regulator. In fig. 3 in the parameter space $Z=\left\{k_{p}, k_{i}, k_{d}\right\}$ The system stability zone is depicted with $P I^{\lambda} D^{\mu}$ regulator for fractional orders $\lambda=0.7, \mu=0.1$. In this figure, the sections of the stability region are represented by planes perpendicular to the coordinate axis. The cross sections are closed shapes whose areas increase as the setting parameter increases.

Similar areas of stability were obtained with other values of fractional orders $\lambda$ and $\mu$.

\section{Conclusions}

On the basis of the D-split method, analytical expressions were obtained, which describe the boundaries of the global region of stability of linear dynamic systems of fractional order of type "input-output" with fractional $P I^{\lambda} D^{\mu}$-regulators. The stability areas are built on the basis of computational experiments in the space of the parameter settings for fractional $P I^{\lambda} D^{\mu}$ - regulators for fixed orders of diereintegrators in the regulator. An appropriate algorithmic software is developed.

\section{References}

1. Podlubny I. (1999), Fractional Differential Equations, Mathematics in Sciences and Engineering, Academic Press. 
2. Tustin A., Allason J.T., Layton J.M., Jakeways R.J. (1958), The design of systems for automatic control of the position of massive object., Proc. Inst. Electr. Eng, 105(C-1), pp. 1-57.

3. Podlubny I. (1999), Fractional-order systems and PID controllers, IEEE Transactions on Automatic Control, 44, pp. 208-214.

4. Butkovskii A.G., Postnov S.S., Postnova E.A. (2013), Fractional integro-differential calculus and its applications in control theory. II. Fractional dynamic systems: simulation and hardware implementation, Automation and telemechanics, 5, pp. 3-34.

5. Uchaikin V.V. (2008), The method of fractional derivatives, Artichoke, Ulyanovsk.

6. Hamamci S.E., Tan N. (2006), Design of PI controllers for achieving time and frequency domain specifications simultaneously, ISA Trans, 45(4), pp. 529-543.

7. Nejjari F., Roux G, Dahhou B, Benhammou A. (1999), Estimation and optimal control design of a biological wastewater treatment process, Mathematics and computers in simulation, 48, pp. 269-280.

8. Lobok O.P., Goncharenko B.M., Sich M.A., Vykhrova L.G. (2017), Simulation of the optimal automatic control of the process of biological treatment of contaminated waters by controllers of fractional order, Collection of Sciences Works of Kirovograd National Technical University - Engineering in agricultural production, branch engineering, automation, 30, p.152-160. 